Cite this: RSC Adv., 2013, 3, 19769

\title{
The growth of porous carbon fibres through in situ vapour deposition
}

\author{
Yi'en Zhou $^{* a}$ and Liang Hong ${ }^{\text {ab }}$
}

Received 28th June 2013

Accepted 16th August 2013

DOI: 10.1039/c3ra43271h

www.rsc.org/advances

This study investigates the transition of carbon flakes to porous carbon fibres (PCF) through vaporisation fragments of polyaromatic hydrocarbons (PAH) and their in situ condensation under the co-gas atmosphere of $\mathrm{N}_{2}$ and $\mathrm{CO}_{2}(\mathrm{v} / \mathrm{v}=1)$, where $\mathrm{CO}_{2}$ plays a vital role in cutting out vaporizable PAH fragments. The polycrystalline PCF exhibit $d$-spacings as large as $17 \AA$ within the mesoporous framework.

As a unique form of porous carbon, porous carbon fibres (PCF) are unlike either carbon nanotubes or granular activated carbons. PCF possess different contours, sizes and most importantly, polycrystalline structures with broad ranges of $d$-spacing values as well as a mesoporous carbon framework. Chemical vapour deposition method has traditionally been used to grow carbon nanotubes ${ }^{1}$ from $\mathrm{CH}_{4}$ or $\mathrm{C}_{2} \mathrm{H}_{2}$ and bamboo-like carbon nanotubes on copper foils from ethanol. ${ }^{2}$

It has been found that fibrous activated carbons exhibit better capabilities than their granular counterparts to entrap condensable vapours, ${ }^{3-6}$ owing to their structural traits of higher aspect ratios as well as interconnected micro- and meso-pores. Whilst long activated carbon fibres are normally produced from acrylic textile fibres and other polymer precursors through lengthy procedures, ${ }^{7}$ short and ultrathin fibres are formed by the electrospinning technique. ${ }^{8-13}$ In order to generate pores in a carbon matrix, carbon dioxide etching is a post carbonization treatment that engraves internal micropores without causing obvious alteration in contour to the fibres formed. ${ }^{14}$ Alternatively, the construction of various mesoporous carbon frameworks has been carried out through the design of polymer precursors, in which using templates is a typical strategy. ${ }^{15-17}$ Contrary to the direct transition from small organic molecules or polymers to fibrous carbons via carbonization and then $\mathrm{CO}_{2}$ etching, this work unveils an alternative transition from an

${ }^{a}$ Dept of Chemical \& Biomolecular Engineering, National University of Singapore, Singapore. E-mail: chezhouy@nus.edu.sg

${ }^{b}$ Institute of Material Research Engineering, Singapore activated carbon flake to a PCF structure undertaken under $\mathrm{CO}_{2}$ and a co-gas atmosphere only. Hence, this transition differs from the transformations of carbon allotropes ${ }^{18-21}$ because the former, in principle, involves convective mass transfer whereas the latter do not.

The initial carbon powder sample was prepared from 2-hydroxyethyl cellulose (HEC) by the method discussed elsewhere. ${ }^{22}$ The resulting carbonaceous powder was then activated at $700{ }^{\circ} \mathrm{C}$ under $\mathrm{CO}_{2}$ for $1 \mathrm{~h}\left(500 \mathrm{~cm}^{3} \mathrm{~min}^{-1}\right)$ and cooled in an $\mathrm{Ar}$ purging stream. The activated carbon powder consisting of irregular and dense carbon flakes (Fig. 1a) was obtained thereafter through proper cleaning in water. The activated carbon powder was then heated in a tubular reactor to $800{ }^{\circ} \mathrm{C}$ under a specific gas stream $\left(50 \mathrm{~cm}^{3} \mathrm{~min}^{-1}\right)$ and this heat treatment (or incubation) lasted for $5 \mathrm{~h}$ (unless otherwise stated) to incubate PCF. The FESEM images (Fig. $1 \mathrm{~b}$ and c) show clumps of the PCF developed under different incubation atmospheres resulting in different contours. The TEM image (Fig. 1d) was taken from a
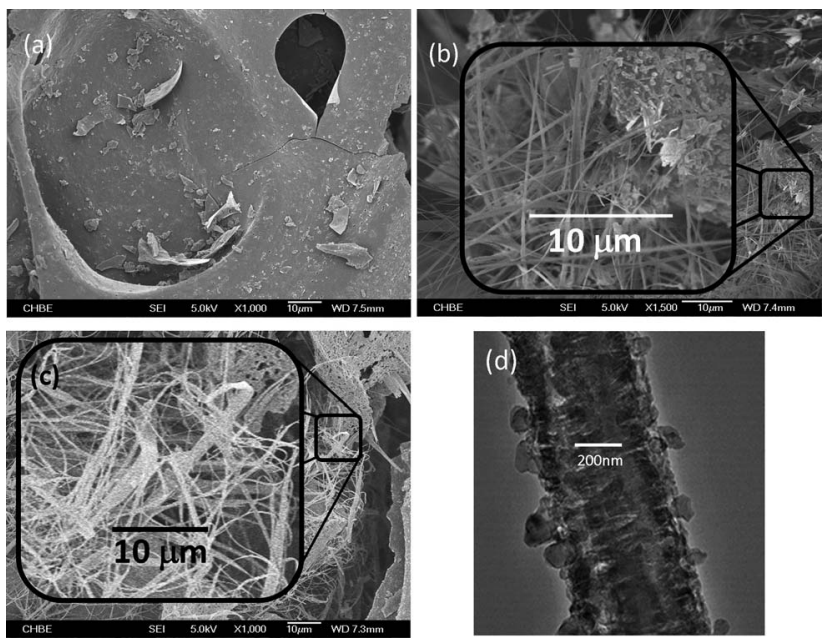

Fig. 1 (a) Activated carbon flake; (b) pure $\mathrm{N}_{2} 5$ h; (c) $\mathrm{N}_{2}-\mathrm{CO}_{2} 5$ h; (d) TEM image of fibre from sample 1 (c). 
strand of the PCF harvested under $\mathrm{N}_{2}-\mathrm{CO}_{2}$. This fibre displays a spine-like structure assembled by sub-micron carbon wedges and interstitial voids. Although the complete mechanism of this transition has yet to be fully understood, experiments done todate including those described in the following sections suggest that it takes place through the vaporization and in situ condensation of PAH fragments leading to the formation of polycrystalline carbon wedges, which subsequently assemble to form the fibres.

The microstructures of the carbon products collated from the reactor were scrutinized by electron microscopy (JSM-6700F Field Emission Scanning Electron Microscope, JEOL) whilst the porous features of the prepared PCF samples were obtained from nitrogen isotherms at $77 \mathrm{~K}$ by Brunauer-Emmett-Teller (BET) surface area measurement (Autosorb-1, Quantachrome instruments). Prior to each measurement, the prepared PCF samples were degassed at $300{ }^{\circ} \mathrm{C}$ for $3 \mathrm{~h}$. The surface area $\left(A_{\mathrm{BET}}\right)$ was calculated from the linear region of the adsorption isotherm under the relative pressure $\left(P / P_{0}\right)$ from 0.05 to 0.3 . Crystalline characteristics of the samples were analysed on a $\mathrm{X}$-ray diffractometer (Brucker $\mathrm{D} 8$ Advance, $\mathrm{Cu} \mathrm{K} \alpha$ radiation $\lambda=1.54 \AA$ ) and the pendant organic functional groups on carbon skeleton were characterized by Fourier transform infrared spectroscopy (FT-IR) using the $\mathrm{KBr}$ pellet window method. Finally, the $\mathrm{H}_{2} \mathrm{~S}$ adsorption attributes of the PCF and the control samples were determined at $25{ }^{\circ} \mathrm{C}$ in a fixed bed microreactor (diameter: $10 \mathrm{~mm}$; length: $200 \mathrm{~mm}$ ) where $0.05 \mathrm{~g}$ of an adsorbent was packed for each measurement. $\mathrm{A}_{2} \mathrm{~S}$ (1100 ppm) $-\mathrm{N}_{2}$ balance stream was fed with a flow rate of $1000 \mathrm{~cm}^{3} \mathrm{~h}^{-1}$ at $1 \mathrm{~atm}$. The $\mathrm{H}_{2} \mathrm{~S}$ concentration in the outlet gas was detected by an electrochemical sensor (MOT500- $\mathrm{H}_{2} \mathrm{~S}$, Keernuo Electronics Technology). The change in $\mathrm{H}_{2} \mathrm{~S}$ concentration in the outlet stream portrays the adsorption capacity of the testing sample. The breakthrough time is defined as the time that elapses before the bed exit-concentration of $\mathrm{H}_{2} \mathrm{~S}$ reaches $1 \%$ of the inlet concentration.

The hypothesis of flake-to-fibre transition through vaporization of $\mathrm{PAH}$ was verified by using a specimen of graphite powder (Sigma Aldrich) in lieu of the carbon flakes to conduct the fibre growth experiment under identical conditions to those used to prepare the sample in Fig. 1c. Unlike the thick PCF growth in sample 1c, only a scarce number of fibres in larger diameters can be incubated from the graphite specimen because of the strong association of graphene sheets in graphite. The occurrence of the fibres supports the etching role of $\mathrm{CO}_{2}$ and the proposed sublimation/deposition mechanism. Fig. 2 provides a closer inspection of the micro-environment surrounding a short segment of an arbitrary fibre originating from graphite. Numerous small graphite chips display discernible rough shapes and emerge near the fibre segment on a graphite chunk. It is thus construed that these chips must be the remaining $\mathrm{CO}_{2}$-reactive graphite pieces from the incubation process. Besides this, the nick in the selected fibre segment shows the bulk morphology of the fibre that comprises tiny strands. These strands were regarded as the product of deposition of the stripped miniature graphene pieces. Accompanying the consecutive fusion of graphene pieces, the strands

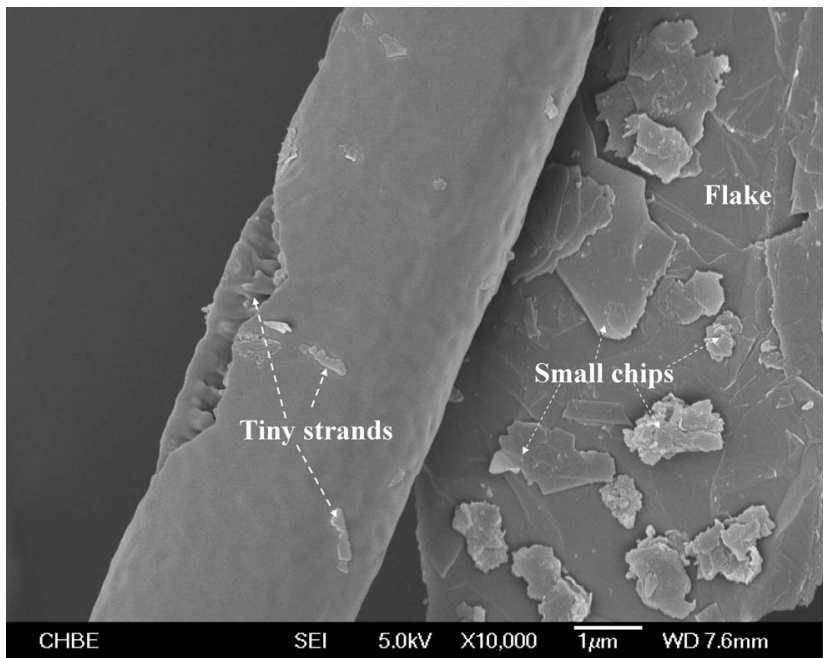

Fig. 2 Magnified view of a single fibre grown from graphite particles attached to its surface under $\mathrm{N}_{2}-\mathrm{CO}_{2}$ incubation.

gradually merged together to form scaffolds, which ultimately connected with each other to form a fibre. As it would be far more difficult to strip graphenes than PAHs from their respective carbon sources under the incubation conditions, much fewer fibres could be eventually generated from graphite. Moreover, unlike what was shown in Fig. 1d, the dense fibre matrix might arise from continuous filling of tiny graphene pieces into niches throughout the course of fibre formation. We also observed that only trace quantities of fibre strands could be generated if $\mathrm{CO}_{2}$ was absent from the incubation atmosphere, validating the role of $\mathrm{CO}_{2}$ in skinning miniature graphenes.

With the exception of the above control experiment, the infrared spectra of the three incubated samples obtained from different treatment conditions (Fig. 3) show clearer aromatic ring stretching peaks (close to $1500 \mathrm{~cm}^{-1}$ and $1600 \mathrm{~cm}^{-1}$ ) compared to that of the activated carbon powder besides the overtone bands (1600-1800 $\left.\mathrm{cm}^{-1}\right)$. This is due to the occurrence

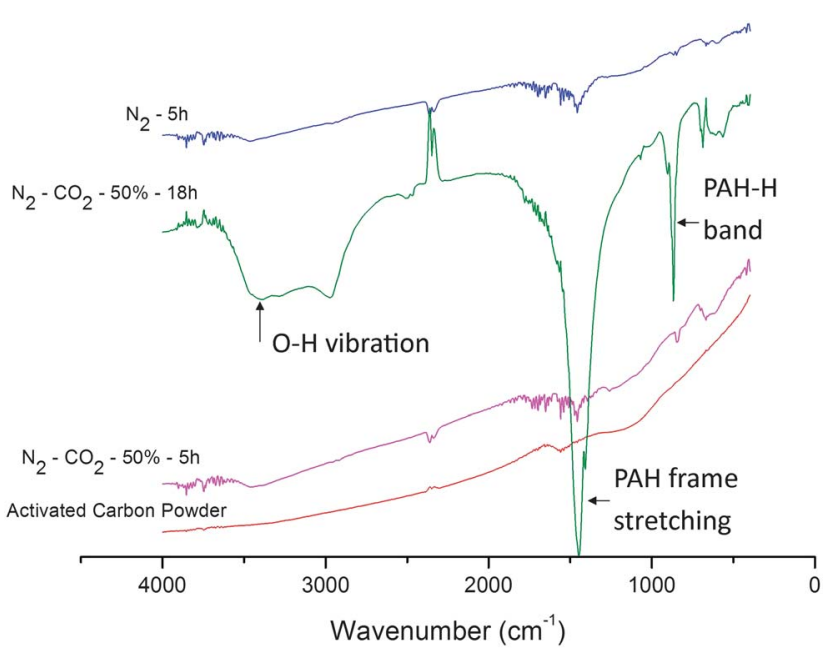

Fig. 3 FT-IR spectra showing the change in organic functional groups due to activation and incubation. 
of a fraction of smaller PAH rings with similar sizes ${ }^{22}$ as the compiling PCF. It is rational to deem that both $\mathrm{CO}_{2}$ and thermal etchings cut up $\mathrm{PAH}$ rings on the surface of flakes into smaller ones, which contribute to the construction of PCF. In particular, an extension of incubation from 5 to $18 \mathrm{~h}$ under $\mathrm{N}_{2}-\mathrm{CO}_{2}$ gave rise to a majority of smaller $\mathrm{PAH}$ species, which also bear phenolic groups according to the obvious $\mathrm{O}-\mathrm{H}$ vibration band $\left(3400 \mathrm{~cm}^{-1}\right)$. Yet, the $18 \mathrm{~h}$ incubation left behind only a small amount of carbon residue, including negligible PCF. The reason for the depletion of PCF from this sample should be due to the fact that PCF is far reactive than the carbon flakes towards $\mathrm{CO}_{2}$. Hence, the growth of PCF and the in situ etching of them shifted to the latter side with the extension of treatment, leaving behind a collection of smaller $\mathrm{PAH}$ with the substituted $-\mathrm{OH}$ group. This functional group must initially come from $\mathrm{CO}_{2}$ through a complex conversion process since the activated carbon sample showed no oxy-group characteristics in its IR spectrum. It can also be deduced that the $\mathrm{PAH}$ with a high $-\mathrm{OH}$ substitution degree is immune to further etching by $\mathrm{CO}_{2}$ and converted to PCF. Therefore, the residue survived from the excessively extended incubation duration.

It is also noteworthy that the activation step had created some reactive sites in the carbon flakes before the incubation treatment. It is essential to the subsequent growth of PCF. This is because the incubation step applied a delicate flow rate of $\mathrm{CO}_{2}$ as described above in order to avert PCF formed from being destroyed by a higher $\mathrm{CO}_{2}$ flow. In addition, the use of $\mathrm{N}_{2}$ instead of $\mathrm{CO}_{2}-\mathrm{N}_{2}$ as the atmosphere for incubation also resulted in PCF as shown in Fig. 1c. This test shows as well the effect of the reactive sites inherited from the previous activation step. It is also clear that the PCF in Fig. 1b display straighter contours and a denser matrix compared to that in Fig. 1c. As per the observation, the thermal etching under $\mathrm{N}_{2}$ should produce $\mathrm{PAH}$ fragments with more regular structure and hence, a more closely packed PCF matrix as a logical conclusion, which is further justified subsequently by BET surface analysis.

The impact of incubation atmosphere on both the conversion and pore structures in the three co-gases $\left(\mathrm{N}_{2}, \mathrm{He}\right.$, or $\left.\mathrm{Ar}\right)$ of $\mathrm{CO}_{2}$ demonstrated that lesser PCF were formed in $\mathrm{CO}_{2}-\mathrm{He}$ compared to $\mathrm{CO}_{2}-\mathrm{N}_{2}$ (Fig. 1c), whilst almost no PCF were obtained (with most of the initial carbon flakes remaining intact) when the transition was conducted in $\mathrm{CO}_{2}-\mathrm{Ar}$. This fact suggests that the co-gas would have affected the activity of $\mathrm{CO}_{2}$. This observation may be explained using the pre-exponential factor $A$ of bi-molecular collision model ${ }^{23}$ since it reflects collision effectiveness at a given temperature:

$$
A=\frac{1}{4} N_{\mathrm{A}} \pi\left(d_{i}+d_{j}\right)^{2}\left[\frac{8 R T}{\pi}\left(\frac{M_{i}+M_{j}}{M_{i} M_{j}}\right)\right]^{\frac{1}{2}} \mathrm{e}^{0.5}
$$

where the subscript $i$ and $j$ represent two molecules; symbol $d$ and $M$ represent molecular diameter and molecular weight respectively. The cross $A$ values show the order $A\left(\mathrm{CO}_{2}-\mathrm{He}\right)>$ $A\left(\mathrm{CO}_{2}-\mathrm{N}_{2}\right)>A\left(\mathrm{CO}_{2}-\mathrm{Ar}\right)$ while the $A$ values of same kind $(i=j)$ are trivial in comparison. In principle, a greater $A$ value will lead to more frequent intermolecular collisions and hence, impede the probability of the gas molecules landing onto carbon surfaces.
On the other hand, the competitive adsorption onto the carbon surface by each individual co-gas against $\mathrm{CO}_{2}(330 \mathrm{pm})$ should follow their kinetic diameters, ${ }^{24}$ which is $\mathrm{N}_{2}(364 \mathrm{pm})>\mathrm{Ar}$ $(340 \mathrm{pm})>\mathrm{He}(260 \mathrm{pm})$. It is therefore considered that the $\mathrm{CO}_{2}-\mathrm{N}_{2}$ combination trades off these two factors, granting $\mathrm{CO}_{2}$ highest touchdown frequency to the carbon flakes. Similarly, a change in the $\mathrm{CO}_{2}$ concentration in $\mathrm{N}_{2}$ from $50 \%$ to either $90 \%$ or $10 \%$ produced fewer fibres due to an over or insufficient etching extent.

During the condensation process, a coaxial stacking of submicron carbon wedges that consists of the planar PAH molecules takes place (Fig. 1d). This hierarchy is verified by the X-ray diffraction results (Fig. 4). Against an amorphous structure displayed by the activated carbon flakes, the products from the incubation under the three different atmospheres (i.e. $\mathrm{N}_{2}, \mathrm{CO}_{2}-\mathrm{N}_{2}$, and $\mathrm{CO}_{2}-\mathrm{He}$ ) display different polycrystalline structures as a result of multiple randomised stacking of PAH molecular fragments different in sizes and with oxy-groups attached. It is noted that the PCF incubated under $\mathrm{N}_{2}$ (or $\mathrm{He}$ )$\mathrm{CO}_{2}$ displays a very broad coverage of $d$-spacing, ranging from $17.9 \AA$ to a mere $0.91 \AA$. Thus, the PCF offer far greater distances between PAH sheets than normal graphitized carbon adsorbents that have the inter-graphene distance of $3.35 \AA^{25}$ As noted above, the residue of the $18 \mathrm{~h}$ incubation under $\mathrm{N}_{2}-\mathrm{CO}_{2}$ is prevalently amorphous phase due to the diminution of the majority of PCF. A conclusion can thus be drawn from the above X-ray characterization: it was the PCF rather than the activated carbon source that contributed to the crystalline features despite the difficulty in separating PCF from the carbon residue as both were entangled together. As such, the intensity of crystalline peaks could approximate the relative content of PCF in a sample. Indeed, similar conversions of the activated carbon flakes to PCF were attained under the $\mathrm{He}-\mathrm{CO}_{2}$ and $\mathrm{N}_{2}-\mathrm{CO}_{2}$ streams, respectively.

Although there was difficulty in separating the PCF from the flakes, close examination under electron microscopy shows that

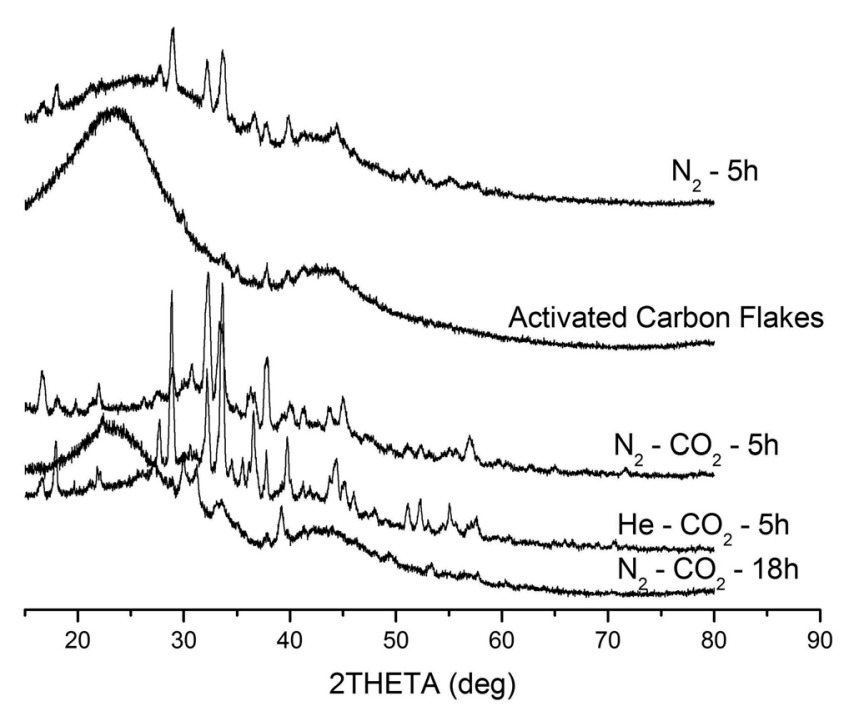

Fig. 4 X-ray diffraction results. 
both the $\mathrm{He}-\mathrm{CO}_{2}$ and $\mathrm{N}_{2}-\mathrm{CO}_{2}$ streams produced a conversion of ca. $40 \%$ to fibres by volume ( $40 \%$ yield) whilst conversion under $\mathrm{Ar}-\mathrm{CO}_{2}$ stream produced no PCF ( $0 \%$ yield). A mass loss of $c a$. $20 \%$ was also observed in all samples regardless of the incubation atmosphere. In addition, as mentioned previously, $\mathrm{CO}_{2}$ plays a major role in this transition and the yield drops significantly to $c a$. $15 \%$ when $\mathrm{CO}_{2}$ was not included in the treatment during the incubation. The incubation duration, the identity of co-gas, and the flow rate of $\mathrm{CO}_{2}$ are the key factors affecting the yield of PCF at the selected incubation temperature, which have been elucidated in the preceding paragraphs.

As far as the porous structures evolved under different atmospheres are concerned, their BET isotherms and the specific surface properties are ostensibly greater than that of the activated carbon flakes specimen, which exhibits a feeble microporous structure with the most abundant pore size of $16 \AA$ $\left(0.24 \mathrm{~cm}^{3} \mathrm{~g}^{-1}\right)$ and a specific surface area of $250 \mathrm{~m}^{2} \mathrm{~g}^{-1}$. When it is incubated under $\mathrm{N}_{2}$, the pure thermal etching triggers formation of mesoporosity $\left(v_{\mathrm{m}} / v_{\mathrm{t}}=0.66\right)$. It is in accordance to its lower $2 \theta$ XRD peaks together with an increase in surface area. Furthermore, a change of the incubation atmosphere to $\mathrm{N}_{2}-\mathrm{CO}_{2}$ permits $\mathrm{CO}_{2}$-etching and hence, resulting in an even greater harvest of PCF. This contributes towards the mesoporosity $\left(v_{\mathrm{m}} / v_{\mathrm{t}}=0.68\right)$, the total specific pore volume $\left(0.81 \mathrm{~cm}^{3} \mathrm{~g}^{-1}\right)$ and the surface area $\left(1140 \mathrm{~m}^{2} \mathrm{~g}^{-1}\right)$. As aforementioned, if the incubation was extended to $18 \mathrm{~h}$ under the same atmosphere, only the phenolic PAH remained and showed a low surface area of $131 \mathrm{~m}^{2} \mathrm{~g}^{-1}$ with a slightly increased mesoporosity $\left(v_{\mathrm{m}} / v_{\mathrm{t}}=0.73\right)$ but a $v_{\mathrm{t}}$ of $0.10 \mathrm{~cm}^{3} \mathrm{~g}^{-1}$. This outcome supports the previous inference for the occurrence of small PAH with similar ring sizes on the basis of the FT-IR spectrum since these $\mathrm{PAH}$ rings favour closely packing. In addition, the use of $\mathrm{He}-$ $\mathrm{CO}_{2}$ as incubation atmosphere instead of $\mathrm{N}_{2}-\mathrm{CO}_{2}$ demonstrated the modest surface properties but a high mesoporosity $\left(v_{\mathrm{m}} / v_{\mathrm{t}}=0.80\right)$. Such effect can be presumed to be caused by the active collisions between $\mathrm{CO}_{2}$ by $\mathrm{He}$ molecules, which made $\mathrm{CO}_{2}$ could adsorb only those reactive carbon sites. With the $\mathrm{CO}_{2}$ etching role, these sites become micro-pores and more preferential to $\mathrm{CO}_{2}$ and hence the micro-pores were expanded to mesopores. The absorption of $\mathrm{H}_{2} \mathrm{~S}$ to the three selected specimens showed different breakthrough durations on the basis of per gram adsorbent: $1.4 \mathrm{~min}$ (AC-flake); $14.2 \mathrm{~min}\left(\mathrm{CO}_{2}-\mathrm{N}_{2}\right)$, $23.4 \mathrm{~min}\left(\mathrm{CO}_{2}-\mathrm{He}\right)$, which is in accord with the volume fraction of mesopores $\left(v_{\mathrm{m}} / v_{\mathrm{t}}\right)$ (Fig. 5).

In conclusion, we have observed and described the phenomenon that a specific type of activated carbon flakes undergoes a sublimation-like process to transform to fibrous structures under a $\mathrm{CO}_{2}$-containing binary atmosphere at $800{ }^{\circ} \mathrm{C}$. This C-to-C transition leads to fibrous activated carbon with sizes between carbon nanotube and carbon fibre and a micro to meso porous structure. The co-gas of $\mathrm{CO}_{2}$ plays a vital role in shaping the evolution of the activated fibre and, in particular, its porous structure. This phenomenon is presumed to originate from the different molecular collision efficiencies and the kinetic diameters of gases. Finally, the $\mathrm{H}_{2} \mathrm{~S}$ adsorption test was undertaken to validate the surface properties obtained from BET analysis.

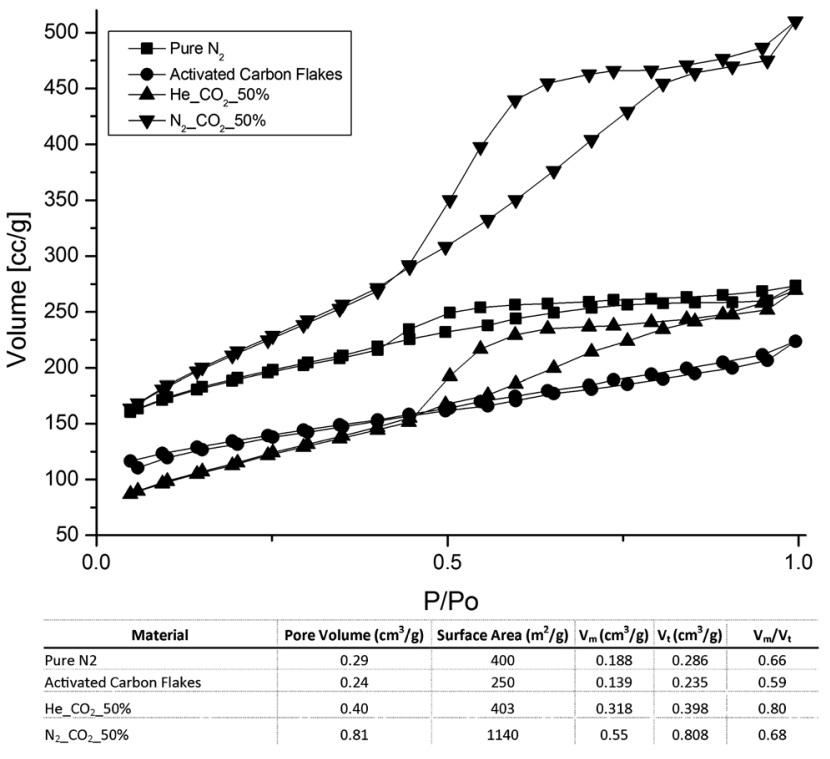

Fig. 5 Isotherms of BET for various pore structures.

The authors would like to thank the National University of Singapore (NUS) and Singapore National Research Foundation (NRF grant no.: R-279-000-261-281) for the support rendered in the course of this research work.

\section{Notes and references}

1 H.-C. Lin, S.-T. Shiue, Y.-H. Cheng, T.-J. Yang, T.-C. Wu and H.-Y. Lin, Carbon, 2007, 45, 2004-2010.

2 J. T. Zhu, J. C. Jia, F. L. Kwong and D. H. L. Ng, Carbon, 2012, 50, 2504-2512.

3 E. A. Dawson, G. M. B. Parkes, P. A. Barnes, M. J. Chinn, L. A. Pears and C. J. Hindmarsh, Carbon, 2002, 40, 28972903.

4 M. Suzuki, Carbon, 1994, 32, 577-586.

5 Y. K. Zhan, C. Y. Nie, H. B. Li, L. K. Pan and Z. Sun, Electrochim. Acta, 2011, 56, 3164-3169.

6 J. Y. Zhou, Z. W. Wang, R. Zuo, Y. Zhou, X. M. Cao and K. Cheng, Asia-Pac. J. Chem. Eng., 2012, 7, S245-S252.

7 P. J. M. Carrott, J. M. V. Nabais, M. M. L. Ribeiro Carrott and J. A. Pajares, Carbon, 2001, 39, 1543-1555.

8 M. Aceituno-Medina, A. Lopez-Rubio, S. Mendoza and J. M. Lagaron, Food Hydrocolloids, 2013, 31, 289-298.

9 S. Agarwal, A. Greiner and J. H. Wendorff, Prog. Polym. Sci., 2013, 38, 963-991.

10 T. Maitra, S. Sharma, A. Srivastava, Y.-K. Cho, M. Madou and A. Sharma, Carbon, 2012, 50, 1753-1761.

11 Y. Qiu, J. Yu, T. Shi, X. Zhou, X. Bai and J. Y. Huang, J. Power Sources, 2011, 196, 9862-9867.

12 X. Song, Z. Wang, Z. Li and C. Wang, J. Colloid Interface Sci., 2008, 327, 388-392.

13 A. Greiner and J. H. Wendorff, Angew. Chem., Int. Ed., 2007, 46, 5670-5703.

14 J. Alcañiz-Monge, D. Cazorla-Amorós, A. Linares-Solano, S. Yoshida and A. Oya, Carbon, 1994, 32, 1277-1283. 
15 D. Gunther, J. Beckmann, M. Schoneich, P. Schmidt and O. Klepel, Carbon, 2012, 50, 3096-3098.

16 S. Lei, J. I. Miyamoto, T. Ohba, H. Kanoh and K. Kaneko, J. Phys. Chem. C, 2007, 111, 2459-2464.

17 Y. Meng, D. Gu, F. Q. Zhang, Y. F. Shi, H. F. Yang, Z. Li, C. Z. Yu, B. Tu and D. Y. Zhao, Angew. Chem., Int. Ed., 2005, 44, 7053-7059.

18 C. He, L. Z. Sun and J. Zhong, Journal of Superhard Materials, 2012, 34, 386-399.

19 F. P. Bundy, W. A. Bassett, M. S. Weathers, R. J. Hemley, H. K. Mao and A. F. Goncharov, Carbon, 1996, 34, 141153.

20 V. V. Brazhkin, A. G. Lyapin, S. V. Popova, S. C. Bayliss, T. D. Varfolomeeva, R. N. Voloshin, A. G. Gavrilyuk,
M. V. Kondrin, V. V. Mukhamad'yarov, I. A. Troyan, S. V. Demishev, A. A. Pronin and N. E. Sluchanko, JETP Lett., 2002, 76, 681-692.

21 K. Yamada, Y. Tanabe and A. B. Sawaoka, Philos. Mag. A, 2000, 80, 1811-1828.

22 M. Sun and L. Hong, Carbon, 2011, 49, 2173-2180.

23 P. W. Atkins, Atkins' physical chemistry, Oxford University Press, Oxford, New York, 2006.

24 G. Aguilar-Armenta, G. Hernandez-Ramirez, E. FloresLoyola, A. Ugarte-Castaneda, R. Silva-Gonzalez, C. TabaresMunoz, A. Jimenez-Lopez and E. Rodriguez-Castellon, J. Phys. Chem. B, 2001, 105, 1313-1319.

25 C. G. Zoski, Handbook of Electrochemistry, Elsevier Science, 2007. 